\title{
A NUMERICAL CHARACTERIZATION OF HYPERSURFACE SINGULARITIES
}

\author{
JUAN ELIAS
}

(Communicated by Louis J. Ratliff, Jr.)

\begin{abstract}
In this note we give a numerical characterization of hypersurface singularities in terms of the normalized Hilbert-Samuel coefficients, and we interpret this result from the point of view of rigid polynomials.
\end{abstract}

\section{INTRODUCTION}

Herrmann and Ikeda proved that for several types of singularities (Buchsbaum of positive depth, homogeneous, and equimultiple), if the minimum among the multiplicities of the hypersurfaces containing the singularity coincides with the multiplicity, then the singularity is a hypersurface [HI].

In this paper we prove that the condition above, plus a condition expressed in terms of the normalized Hilbert-Samuel coefficients, give a characterization of the hypersurface singularities, Theorem 2.1 ; we prove this result without any hypothesis on the singularity. We end this paper interpreting the characterization of the hypersurface singularities from the point of view of rigid polynomials, Corollary 2.2.

\section{ChARACTERIZATION OF HYPERSURFACE SINGULARITIES}

Let $R$ be the power series ring $\mathbf{k}\left[\left[X_{1}, \ldots, X_{N}\right]\right]$, where $\mathbf{k}$ is an infinite field. Let $I \subset R$ be a proper ideal of $R$; we denote by $s(I)$ the order of $I$ and by $e_{0}(A), \ldots, e_{d}(A)$ the normalized Hilbert-Samuel coefficients of $A=R / I$.

Let $x=\left\{x_{1}, \ldots, x_{t}\right\}$ be a set of elements of $A$ such that $x_{i}$ is a degree one superficial element of $A /\left(x_{1}, \ldots, x_{i-1}\right)$ for all $i=1, \ldots, t$ [ZS]; we say that $x$ is a superficial sequence of $A$ of length $t$. We define $l\left(x_{0}\right)=$ length $\left(\left(x_{1}, \ldots, x_{t-1}\right): x_{t} /\left(x_{1}, \ldots, x_{t-1}\right)\right)$; if $A$ is $d$-dimensional, $d \geq 2$, then we put $l(A)=\operatorname{Min}\left\{l\left(x_{0}\right) \mid x\right.$. superficial sequence of length $d-1$ of $\left.A\right\}$. We put $l(A)=0$ for $d=1$. Since $\mathbf{k}$ is infinite there exists a nonempty Zariski open set $U(A)$ of the $N(d-1)$-dimensional affine space over $\mathbf{k}$, parameterizing the sets of $d-1$ linear forms of $R$ such that: for all $\left(L_{1}, \ldots, L_{d-1}\right) \in U(A)$, the

Received by the editors April 18, 1991 and, in revised form, October 9, 1991 and January 16. 1992.

1991 Mathematics Subject Classification. Primary 14B05; Secondary 13H15.

Partially supported by DGICYT PB88-0224. 
cosets in $A$ of $L_{1}, \ldots, L_{d-1}$ are a superficial sequence of length $d-1$ and $s(I)=s\left(I+\left(x_{\bullet}\right) /\left(x_{\bullet}\right)\right)$.

For every pair of positive integers $(b, e)$ we define $\rho_{0, b, e}=(r+1) e-\left(\begin{array}{c}r+b \\ r\end{array}\right)$ where $r$ is the integer such that $\left(\begin{array}{c}b+r-1 \\ r\end{array}\right) \leq e<\left(\begin{array}{c}b+r \\ r+1\end{array}\right)$, and we put $\rho_{1, b, e}=$ $e(e-1) / 2-(b-1)(b-2) / 2$.

Theorem 2.1. Let $A=R / I$ be a d-dimensional, $d \geq 1$, local ring with embedding dimension $b \geq 2$. Assume that $e_{0}(A)=s(I) \geq 2$. The following conditions are equivalent:

(1) $e_{1}(A) \geq l(A)+\rho_{0, b-d+1, e}-\rho_{1, b-d+1, e}$, with $e=e_{0}(A)$.

(2) There exists $F \in M^{s} \backslash M^{s+1}$, such that $I=(F)$ with $s=s(I)$.

Proof. If (2) holds then $A$ is Cohen-Macaulay, so $l(A)=0$. Since $e_{1}(A) \geq 0$ and $0 \geq \rho_{0, b-d+1, e}-\rho_{1, b-d+1, e}[\mathrm{E}]$, we deduce $(1)$.

Assume $d=1$. Condition (1) says $e_{1}(A) \geq \rho_{0, b, e}-\rho_{1, b, e}$. Let $B$ denote the associated graded ring to $A$, and let $J$ be intersection of the minimal primary components of 0 in $B$. Since $B / J$ is a one-dimensional graded CohenMacaulay ring of multiplicity $e$, for all $n \geq e-1$ we have $\operatorname{dim}_{\mathbf{k}}\left((B / J)_{n}\right) \geq e$ [M, Proposition 12.10]. Hence for all $n \geq e-1$ we $\operatorname{deduce} \operatorname{dim}_{\mathbf{k}}\left(B_{n}\right) \geq e$. From the assumption $e=s(I)$ we obtain $\operatorname{dim}_{\mathbf{k}}\left(B_{n}\right)=\left(\begin{array}{c}b-1+n \\ b-1\end{array}\right)$ for $n \leq e-1$. Hence we have for $n \gg 0$

$$
e n-e_{1}(A)=\operatorname{length}_{A}\left(A / m^{n}\right) \geq e n-\rho_{0, b, e}+\left(\begin{array}{c}
b+e-2 \\
b-1
\end{array}\right)-e,
$$

$m$ is the maximal ideal of $A$, and we deduce $\rho_{0, b, e}-\rho_{1, b, e} \leq e_{1}(A) \leq$ $\rho_{0, b, e}-\left(\begin{array}{c}b+e-2 \\ b-1\end{array}\right)+e$.

From this we obtain $0 \leq \rho_{1, b, e}-\left(\begin{array}{c}b+e-2 \\ b-1\end{array}\right)+e$. Note that the right-hand side of the inequality is a decreasing function of $b$ and is negative for $b=3$, so we deduce $b=2$.

Assume that $d \geq 2$. For all superficial sequence $x . \in U(A)$ the following holds: $\operatorname{dim}\left(A /\left(x_{\bullet}\right)\right)=1, b\left(A /\left(x_{\bullet}\right)\right)=b-(d-1), e=e_{0}\left(A /\left(x_{\bullet}\right)\right)=$ $s\left(I+\left(x_{\bullet}\right) /\left(x_{\bullet}\right)\right) \geq 2$, and $e_{1}\left(A /\left(x_{\bullet}\right)\right)=e_{1}(A)-l(A) \geq \rho_{0, b-d+1, e}-\rho_{1, b-d+1, e}$; so from the case $d=1$ we obtain $b-d+1=2$. Hence we have proved that for all $d \geq 1, b=d+1$ holds, and then $\operatorname{ht}(I)=1$. Let $J=(F)$ be the intersection of the minimal primary components of $I$ of height 1 . Note that

$$
\operatorname{order}(F)=e_{0}(R /(F))=e_{0}(A)=s(I) ;
$$

from $I \subset(F)$ we deduce $I=(F)$.

\section{INTERPRETATION IN TERMS OF RIGIDITY}

Definition [E]. Let $\mathscr{C}$ be a set of local rings. We say that a polynomial $p(T) \in$ $\mathbb{Q}[T]$ is rigid for $\mathscr{C}$ if there exists a numerical function $F_{p}: \mathbf{N} \rightarrow \mathbf{N}$ such that for all $A \in \mathscr{C}$ with Hilbert-Samuel polynomial $p$ we have the Hilbert-Samuel function is $F_{p}$, i.e., the Hilbert-Samuel polynomial determines the HilbertSamuel function.

For the basic properties of rigid polynomials see [E, EV, S]. 
Corollary 2.2. Let $\mathscr{C}$ be the set of local rings $A=R / I$ with $l(A) \leq l$, embedding dimension $b$, and such that $e_{0}(A)=s(I) \geq 2$. Then every polynomial $p(T)=$ $\sum_{i=0}^{d}(-1)^{i} e_{i}\left(\begin{array}{c}T+d-1-i \\ d-i\end{array}\right)$ with $e_{1} \geq l+\rho_{0, b-d+1, e_{0}}=\rho_{1, b-d+1, e_{0}}$ is rigid for $\mathscr{C}$; the associated Hilbert-Samuel function is $F_{p}(n)=\left(\begin{array}{c}b+n-1 \\ b-1\end{array}\right)-\left(\begin{array}{c}b+n-1-e_{0} \\ b-1\end{array}\right)$.

\section{REFERENCES}

[E] J. Elias, Characterization of the Hilbert-Samuel polynomials of curve singularities, Compositio Math. (2) 74 (1990), 135-155.

[EV] J. Elias and G. Valla, Rigid Hilbert functions, J. Pure Appl. Algebra 71 (1991), 19-41.

[HI] M. Herrmann and S. Ikeda, Three notes on the order of ideals defining hypersurfaces, J. Algebra 132 (1990), 123-129.

[M] E. Matlis, 1-dimensional Cohen-Macaulay rings, Lecture Notes in Math., vol. 327, SpringerVerlag, Berlin and New York, 1977.

[S] J. Sally, Rigid Hilbert polynomials for m-primary ideals, preprint, 1990.

[ZS] O. Zariski and P. Samuel, Commutative algebra. II, van Nostrand, New York, 1958.

Departmento Algebra i Geometria, Universitat de Barcelona, Gran Via 585, 08007 Barcelona, Spain

E-mail address: UBAAGE01@EBCESCA1 